\title{
含氟共轭微孔聚合物的制备及其吸附应用研究
}

\author{
岳勇 钱祉祺 孔凡安 肖琴 任世杰* \\ (四川大学 高分子科学与工程学院 高分子材料工程国家重点实验室 成都 610065)
}

\begin{abstract}
摘要 共轭微孔聚合物由于其高的比表面积、优良的物理化学稳定性、多样的合成方法以及沿分子骨架延伸的共轭结 构等特点, 近几年得到广泛关注和快速发展. 本工作以 1,3,5-三氟-2,4,6-三碘苯作为含氟单体与 1,3,5-三乙炔基苯通过 Sonogashira 偶联反应聚合得到含氟共轭微孔聚合物 F-CMP. 通过把氟原子引入到共轭微孔聚合物骨架中, F-CMP 显示 出良好的疏水性能, 与水的接触角达到 $145^{\circ}$. 得益于良好的疏水性能和适宜的孔隙结构, 相比于骨架结构相似的不含 氟共轭微孔聚合物(H-CMP), F-CMP 对油和有机溶剂的吸附量得到大幅提高, 且显示出高的吸附速率和良好的吸附循 环性.
\end{abstract}

关键词 共轭微孔聚合物; 氟掺杂; 吸附材料

\section{Preparation and Adsorption Application Study of Fluorine-containing Conjugated Microporous Polymers}

\author{
Yue, Yong Qian, Zhiqi Kong, Fanan Xiao, Qin Ren, Shijie* \\ (College of Polymer Science and Engineering, State Key Laboratory of Polymer Materials Engineering, Sichuan University, \\ Chengdu 610065, China)
}

\begin{abstract}
Water pollution arising from ever-growing domestic sewage and industrial organic pollutants has caused severe environmental and ecological problems in many parts of the world. It is urgent to seek appropriate ways to resolve oily wastewater and organic solvent pollution. Currently, physical adsorption is considered to be one of the most important methods to eliminate the oil contaminations in water thanks to its high efficiency and low cost. However, traditional adsorbent materials, such as activated carbon, zeolite and natural fibers, often suffer from low adsorption capacities, poor adsorption selectivity and recyclability. Thus, it is still of great importance to develop new absorbent materials for the separation and removal of oils or organic pollutants from water to address environmental issues. Conjugated microporous polymers (CMPs) are a class of organic porous polymers that have attracted extensive attention thanks to their large specific surface area, good physicochemical stability and unique extended $\pi$-conjugation along the polymer skeleton. Here we report a fluorine-containing conjugated microporous polymer (F-CMP), which was synthesized via Sonogashira cross-coupling reaction from 1,3,5-trifluoro-2,4,6-triiodobenzene and 1,3,5-triethynylbenzene. As a comparison, fluorine-free conjugated microporous polymer (H-CMP) was synthesized in the same condition from 1,3,5-tribromobenzene and 1,3,5-triethynybenzene. By introducing fluorine atom into the conjugated microporous skeleton, the contact angle of F-CMP with water reaches $145^{\circ}$, exhibiting excellent hydrophobicity. Nitrogen adsorption/desorption isotherms of the F-CMP show a high specific surface area of $638 \mathrm{~m}^{2} \cdot \mathrm{g}^{-1}$, and the pore size distribution analysis shows the existence of both micropores and macropores. It can be obtained by adsorption experiments of oil and organic solvents that the adsorption capability of F-CMP increases significantly compared with its fluorine-free counterpart with similar structural skeleton. Due to high hydrophobicity and porous properties, F-CMP shows excellent adsorption properties towards to the removal of organic solvents and oils. The adsorption capability of F-CMP towards pump oil and chloroform can reach $40 \mathrm{~g} / \mathrm{g}$ and $43 \mathrm{~g} / \mathrm{g}$, respectively. Meanwhile, F-CMP shows rapid adsorption rate and excellent adsorption recyclability. Thus, F-CMP displays promising application prospects in the field of organic pollutant adsorption and environmental remediation.
\end{abstract}

Keywords conjugated microporous polymer; fluorine doping; adsorption material

\section{1 引言}

随着经济的快速发展, 生活污水和工业废水急剧增 加, 导致水中废弃油和有机溶剂等污染物的含量逐年上
升 $^{[1]}$. 为了减轻环境的压力, 物理吸附、化学分解和生物 降解等方法被广泛应用到水系污染物的治理中 ${ }^{[2,3]}$. 其 中, 物理吸附由于可以避免对环境的二次污染，且具有 高效率、低成本等优点, 在对废弃油和有机溶剂等污染

\footnotetext{
*E-mail: rensj@scu.edu.cn
}

Received February 22, 2019; published April 17, 2019.

Supporting information for this article is available free of charge via the Internet at http://sioc-journal.cn.

Project supported by the National Natural Science Foundation of China (Nos. 21574087, 21404074) and the State Key Laboratory of Polymer Materials Engineering (No. Sklpme2018-2-05).

项目受国家自然科学基金(Nos. 21574087, 21404074)和高分子材料工程国家重点实验室自主课题(Sklpme2018-2-05)资助. 
物的处理上扮演着重要角色 ${ }^{[4]}$. 然而, 传统的物理吸附 剂, 如活性炭 ${ }^{[5]}$ 、沸石 ${ }^{[6]}$ 和天然纤维 ${ }^{[7]}$ 等, 对污染物往往 表现出吸附量低、选择性吸附和循环性差等缺点. 为了 克服这些不足, 许多新型吸附材料也逐渐应用到污染物 的处理中, 其中包括纳米结构的金属氧化物 ${ }^{[8,9] 、}$ 碳纳米 管 ${ }^{[10]}$ 、多孔石墨烯 ${ }^{[11]}$ 和多孔 $\mathrm{BN}$ 纳米片层 ${ }^{[12]}$ 等, 这些新 材料对污染物表现出良好的吸附性能. 但是, 这些材料 仍然存在制备困难、孔径难以调节以及杂原子引入难以 控制等问题. 因此, 发展具备高吸附量、出色的吸附选 择性和循环性以及低成本的新型物理吸附材料显得尤 为重要.

有机多孔聚合物由于其丰富的孔道结构和多样的 合成方法, 在近几年得到快速地发展 ${ }^{[13 \sim 17]}$. 共轭微孔聚 合物(CMPs)作为有机多孔聚合物的一种, 具有高比表 面积、出色的化学和热稳定性以及沿分子链延伸的共轭 结构等优点 ${ }^{[18]}$, 自 2007 年首次报道以来 ${ }^{[19]}$ 便得到了研 究者的持续关注. 共轭微孔聚合物(CMPs)的这些优点 使其广泛应用于气体吸附 ${ }^{[20]}$ 、催化 ${ }^{[21 ~ 23] 、 \text { 能量储存 }}{ }^{[24,25]}$ 等领域. 为了进一步拓宽共轭微孔聚合物的应用范围, 大量的研究工作将 $\mathrm{N}^{[26]} 、 \mathrm{~S}^{[27]} 、 \mathrm{~B}^{[28]} 、 \mathrm{~F}^{[29]}$ 等杂原子引入 到聚合物骨架中来改善材料的相关性能 ${ }^{[24,30,31]}$. 其中, 把氟元素引入到共轭微孔材料的骨架中可以有效地提 高疏水性 ${ }^{[13,29,32]}$, 为水处理中选择性吸附有机溶剂和废 弃油提供了新的方法.

本文中, 我们选用含氟单体 1,3,5-三氟-2,4,6-三碘 苯与 $1,3,5$-三乙炔苯为结构单元, 通过 Sonogashira 偶联 反应合成了含氟共轭微孔聚合物 F-CMP. 由于氟原子的
引入, F-CMP 与水的接触角达到 $145^{\circ}$, 显示出优异的疏 水性能. 得益于良好的疏水性能和合理的孔隙结构, F-CMP 在对油和有机溶剂的吸附实验中, 表现出大的 吸附量、良好的吸附选择性、快速的吸附速率和出色的 循环性.

\section{2 结果与讨论}

\section{1 聚合物的合成及化学结构表征}

图 1 为两种聚合物的合成路线, 以 $1,3,5$-三乙炔基 苯(M1)与 1,3,5-三氟-2,4,6-三碘苯(M2)和 1,3,5-三溴苯 (M3)反应分别制备了 F-CMP 与 H-CMP. 图 2a 为聚合物 F-CMP 及单体的红外吸收光谱图. 聚合物谱图在 1552 $\mathrm{cm}^{-1}$ 和 $1405 \mathrm{~cm}^{-1}$ 处出现苯环的特征吸收峰, 在 2210 $\mathrm{cm}^{-1}$ 和 $1005 \mathrm{~cm}^{-1}$ 处分别对应 $\mathrm{C} \equiv \mathrm{C}$ 和 $\mathrm{C}-\mathrm{F}$ 的伸缩振 动峰, 证明了聚合物 F-CMP 化学结构. 此外, 同单体 (M1)相比, F-CMP 在 $3280 \mathrm{~cm}^{-1}$ 处没有出现强的端炔 $\mathrm{C}$ - $\mathrm{H}$ 伸缩振动峰, 表明了 Sonogashira 偶联反应的发生. 图 S1 为聚合物 H-CMP 和单体的红外吸收光谱图. 聚合 物谱图中苯环特征峰和 $\mathrm{C} \equiv \mathrm{C}$ 的伸缩振动峰证实了聚合 物 H-CMP 的化学结构.

图 $2 \mathrm{~b}$ 为两个聚合物的 $\mathrm{X}$ 射线光电子能谱图(XPS), 图中峰位置主要位于 $284.8 \mathrm{eV} 、 532 \mathrm{eV}$ 和 $686 \mathrm{eV}$ 处, 分 别对应于 C $1 \mathrm{~s} 、 \mathrm{O} 1 \mathrm{~s}$ 和 F $1 \mathrm{~s}$ 轨道. 聚合物中的氧元素可 能来自于材料孔道中吸附的含氧分子以及在后处理过 程中部分未反应的端炔被氧化 ${ }^{[33]}$. 从 XPS 的元素分析 结果可以看出(表 S1), 相比于 H-CMP, F-CMP 在含有碳

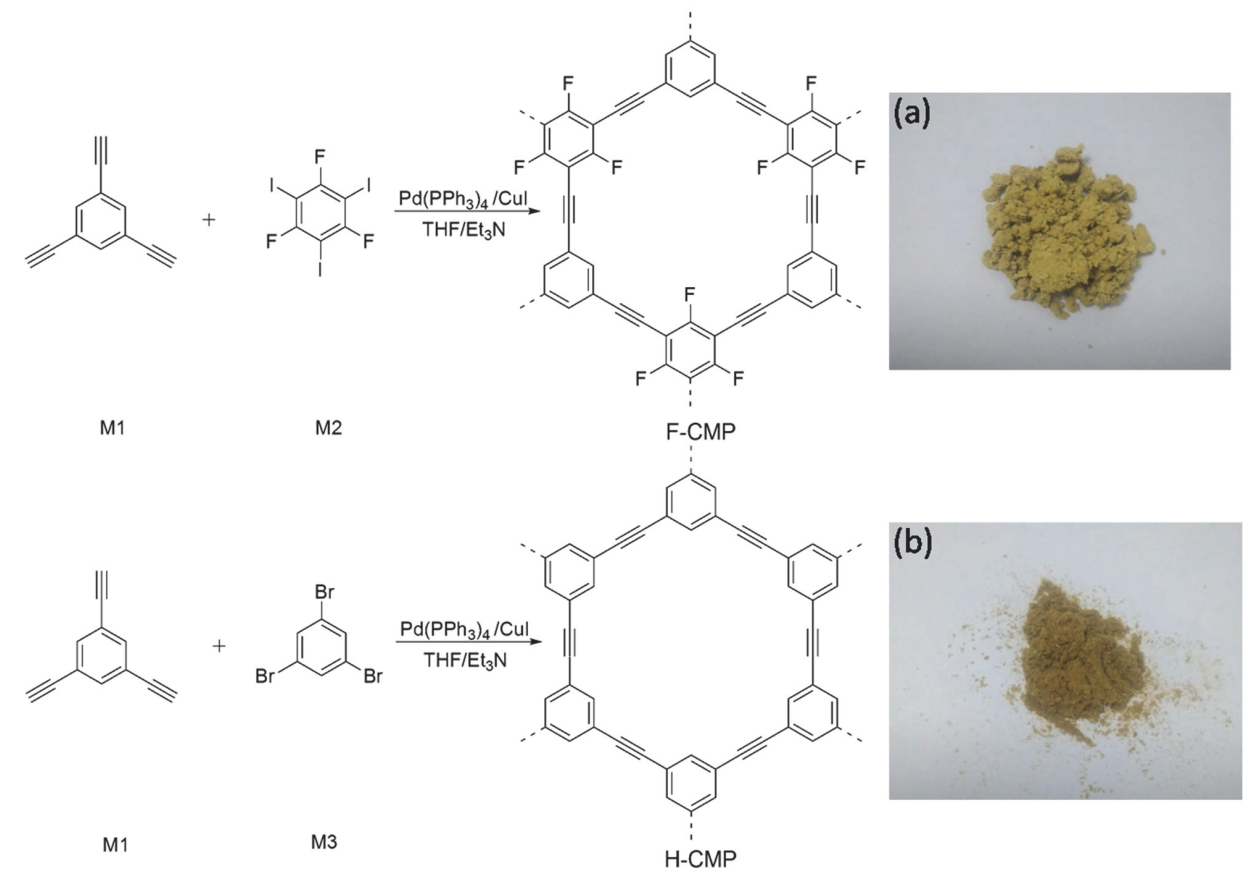

图 1 聚合物 F-CMP 和 H-CMP 的合成步骤; M1: 1,3,5-三乙炔苯, M2: 1,3,5-三氟-2,4,6-三碘苯, M3: 1,3,5-三澳苯; F-CMP 的聚合物光学照片 (a), $\mathrm{H}-\mathrm{CMP}$ 的聚合物光学照片(b)

Figure 1 Synthetic routes of F-CMP and H-CMP; M1: 1,3,5-triethynylbenzene, M2: 1,3,5-Trifluoro-2,4,6-triiodobenzene, M3: 1,3,5-tribromobenzene; photo of F-CMP (a), photo of H-CMP (b) 

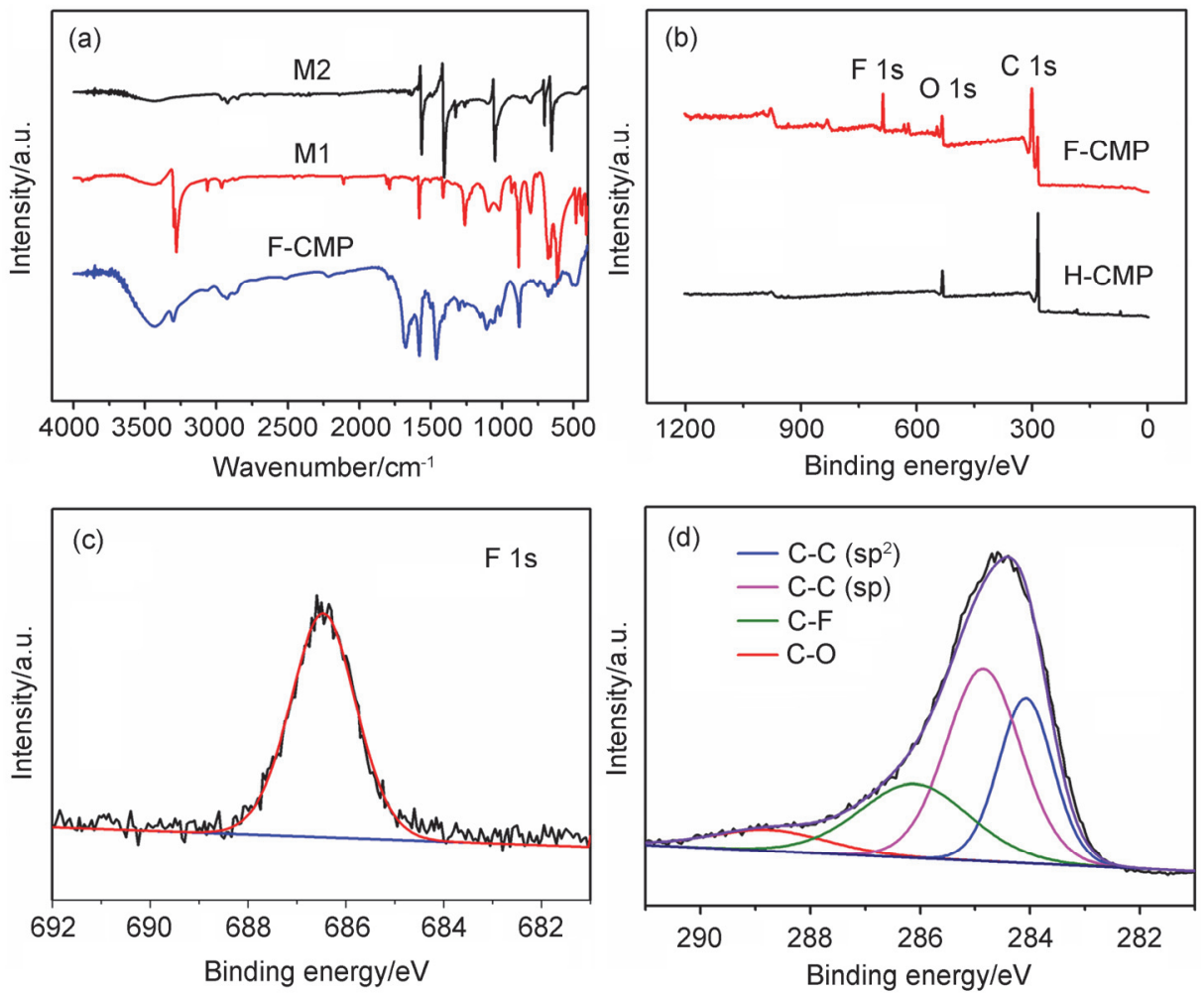

图 2 聚合物 F-CMP 和单体的红外光谱(a), 聚合物的 XPS 全谱图(b), F-CMP 的 F 1s 分谱图(c), F-CMP 的 C 1s 分谱图(d)

Figure 2 FT-IR spectra of F-CMP and the monomers (a), XPS full spectrum survey of the polymers (b), F 1s spectrum of F-CMP (c), C 1s spectrum of F-CMP (d)

和氧的同时，氟元素的含量也达到 $11.74 \mathrm{wt} \%$, 证实了 聚合物中氟元素的成功引入. F-CMP 中氟含量比理论值 低，可能是因为在过渡金属钯的催化下，聚合过程中部 分缺陷处的碳氟键断裂, 氟元素以氟负离子的形式离 去 ${ }^{[34]}$. 对 F-CMP 中 F 1s 进行分峰处理(图 2c), 谱图中出 现单一的 $\mathrm{C}-\mathrm{F}$ 键的峰, 与聚合物中氟的存在形式相印 证. 图 $2 \mathrm{~d}$ 为 F-CMP 的 $\mathrm{C} 1 \mathrm{~s}$ 分峰结果, 其中碳元素主要 以 4 种形式存在, 即 $\mathrm{C}-\mathrm{C}\left(\mathrm{sp}^{2}\right) 、 \mathrm{C}-\mathrm{C}(\mathrm{sp}) 、 \mathrm{C}-\mathrm{F}$ 和 $\mathrm{C}-\mathrm{O}$, 其分别对应的结合能为 $284.1 \mathrm{eV} 、 284.8 \mathrm{eV}$ 、

$286.2 \mathrm{eV}$ 和 $288.8 \mathrm{eV}^{[35]}$. XPS 的结果有力地证明了聚合 物的化学组成和成键形式. 图 S2 为样品的粉末 $\mathrm{X}$ 射线 衍射(XRD)测试结果, 谱图在 $22^{\circ}$ 左右呈现包峰, 说明聚 合物 H-CMP 和 F-CMP 都为非晶材料.

\section{2 聚合物形貌及多孔性能表征}

共轭微孔聚合物聚合反应过程中, 单体的浓度和机 械搅拌速率对聚合物的形貌有很大的影响, 在低单体浓 度下 $(<0.1 \mathrm{~mol} / \mathrm{L})$, 由于单体分子在溶液中良好的分散 性, 所形成的聚合物形貌倾向于管状, 而高单体浓度下 聚合物为纳米颗粒堆积 ${ }^{[36]}$. HCMP 和 FCMP 的聚合均采 用了低单体浓度, 如扫描电子显微镜图 $3 \mathrm{a} \sim 3 \mathrm{~d}$ 所示, H-CMP 与 F-CMP 材料的形貌都是管状的, 且管的直径 分别在 $2.15 \mu \mathrm{m}$ 和 $0.25 \mu \mathrm{m}$. 通过对材料进一步放大观 察, 可以发现 F-CMP 是中空的管状结构, 这种中空的小 纳米管可以让油和有机溶剂更好的进入, 增大与聚合物
材料的接触面.

为进一步分析材料的孔结构和吸附性能, 在 $77 \mathrm{~K}$ 的条件下对材料进行氮气吸脱附实验 ${ }^{[2]}$. 图 3e 中聚合 物表现出 I 型吸附曲线特性, 说明聚合物中均存在大量 的微孔结构. 同时, 吸附曲线在相对压力较高的区域 $\left(p / p_{0}>0.9\right)$, 对氮气的吸附也有明显的上升, 表明聚合 物材料中存在因边缘相互堆叠和内部缺陷而形成的介 孔和大孔结构. 聚合物的孔尺寸分布通过非定域密度泛 函理论(NLDFT)模型获得(图 3f), H-CMP 和 F-CMP 拥有 相似的骨架结构, 其孔径都主要分布在 $0.85 \mathrm{~nm}$ 的微孔 区域. 根据 Brunauer-Emmet-Teller (BET)算法计算出聚 合物 H-CMP 与 F-CMP 的比表面积分别为 $1135 \mathrm{~m}^{2} \cdot \mathrm{g}^{-1}$ 和 $638 \mathrm{~m}^{2} \cdot \mathrm{g}^{-1}$. 多孔材料的微孔率可以用微孔体积与全 孔体积的比例 $\left(V_{\text {micro }} / V_{\mathrm{tot}}\right)$ 来表示, 如表 1 所示, H-CMP 的 微孔率为 $85 \%$, 高于 F-CMP 的 $61 \%$. F-CMP 中含有更多 的介孔和大孔结构, 这些结构可以让有机溶剂和油在材 料中运动阻力变小, 与材料的接触面变大.

表 1 聚合物的比表面积和多孔性质

Table 1 BET surface areas and porosity data of the polymers

\begin{tabular}{ccccc}
\hline Sample & $S A_{\mathrm{BET}}{ }^{a} /\left(\mathrm{m}^{2} \cdot \mathrm{g}^{-1}\right)$ & $V_{\text {micro }}{ }^{b} /\left(\mathrm{m}^{3} \cdot \mathrm{g}^{-1}\right)$ & $V_{\text {tot }}{ }^{c} /\left(\mathrm{m}^{3} \cdot \mathrm{g}^{-1}\right)$ & $V_{\text {micro }} / V_{\text {tot }}$ \\
\hline F-CMP & 638 & 0.26 & 0.43 & 0.61 \\
H-CMP & 1153 & 0.51 & 0.60 & 0.85 \\
\hline
\end{tabular}

${ }^{a}$ Surface area determined by the BET method. ${ }^{b}$ Determined by t-plot method. ${ }^{c}$ Total pore volume calculated at $p / p_{0}=0.99$. 
(a)

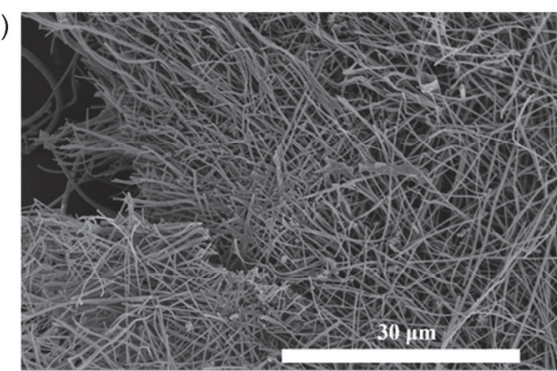

(c)
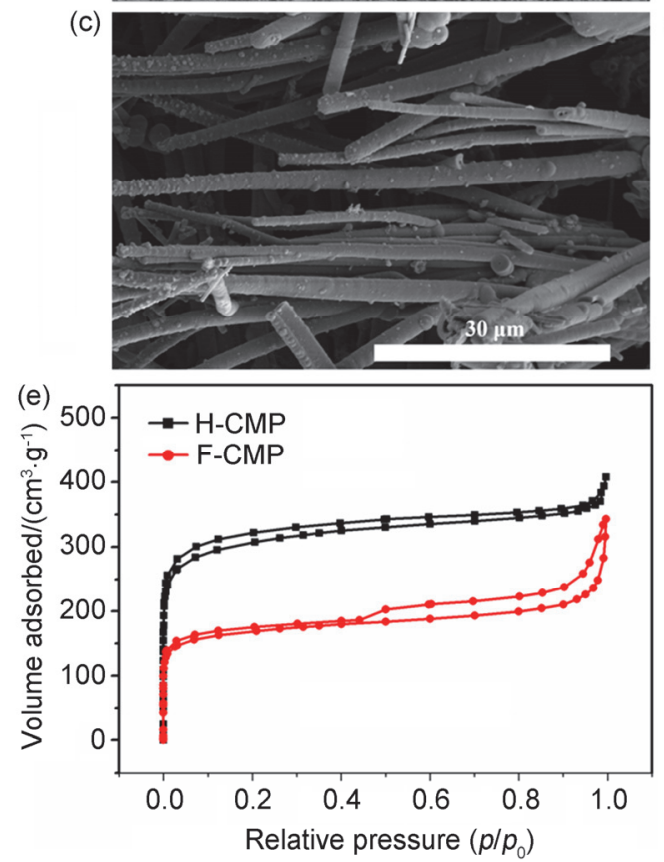

(b)

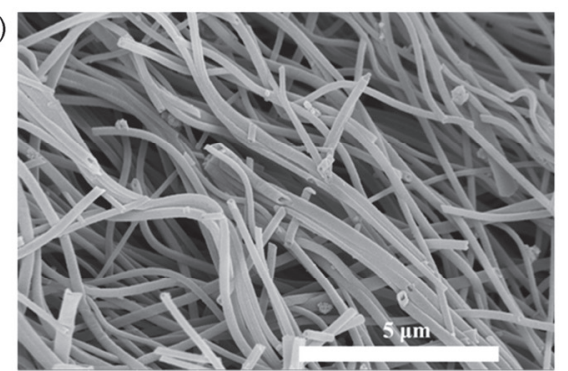

(d)
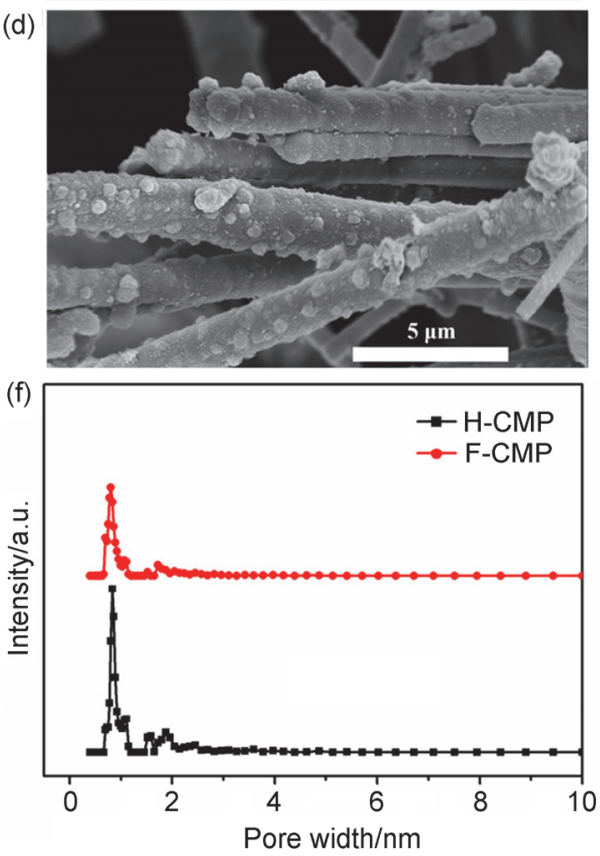

图 3 F-CMP 的扫描电镜图(a)和(b), H-CMP 的扫描电镜图(c)和(d), 聚合物的 $\mathrm{N}_{2}$ 吸附-脱附等温曲线(e)和孔径分布曲线(f)

Figure 3 SEM images of F-CMP (a and b), SEM images of H-CMP (c and d), $\mathrm{N}_{2}$ adsorption-desorption isotherms (e) and pore size distribution curves (f) of the polymers

图 S3 为聚合物在氮气氛围下的热失重曲线. 聚合 物 F-CMP 和 H-CMP 在达到 10\%质量损失时的温度分别 为 $393{ }^{\circ} \mathrm{C}$ 和 $380{ }^{\circ} \mathrm{C}$, 而且在温度达到 $800{ }^{\circ} \mathrm{C}$ 时, 两个 聚合物都有 $73 \%$ 的质量保留, 表明两种聚合物都有良好 的热稳定性. 为了判断材料的亲疏水性能, 我们将聚合 物压片后测试与水的接触角 (图 S4) ${ }^{[37]}$, F-CMP 与 $\mathrm{H}-\mathrm{CMP}$ 的接触角分别为 $145^{\circ}$ 和 $119^{\circ}$, 说明把氟元素引 入到多孔材料中可以有效地提高材料的疏水性 ${ }^{[29]}$. F-CMP 表现出的良好疏水性, 将有利于对油和有机溶 剂的吸附，提高材料的吸附选择性.

\section{3 聚合物吸附性能测试}

F-CMP 材料具有良好的疏水性和合理的孔隙结构, 使其可以对油和有机溶剂进行选择性吸附. 材料的吸附 过程可以从图 $4 \mathrm{a} \sim 4 \mathrm{c}$ 看出, 把用苏丹III染色的甲苯滴 到含 $20 \mathrm{~mL}$ 水的培养血中, 随后在甲苯旁边放入块状的 F-CMP 后开始吸附. 经过 $30 \mathrm{~s}$ 的时间, 我们发现橙红色 甲苯液滴完全消失, 吸附完成后块状 F-CMP 依然漂浮 在水面, 说明 F-CMP 拥有快速的吸附速率和优异的吸 附选择性.
吸附量的大小是衡量材料吸附能力的重要指标之 一. 为了测试材料对油的吸附量, 我们选择泵油和植物 油这类常见的油类进行吸附实验, 吸附量的大小是通过 聚合物吸附前后的质量改变来确定的(图 S5). 图 4d 中 可以看出 F-CMP 对泵油的吸附量达到 $40 \mathrm{~g} / \mathrm{g}$, 为 H-CMP 的吸附量 $(17 \mathrm{~g} / \mathrm{g})$ 的 2.35 倍, 表现出更加优异的 吸附性能. 同时我们选用 DMF、甲醇、正己烷、甲苯、 氯仿和丙酮作为溶剂, 测试了聚合物对不同有机溶剂的 吸附量. 与吸油实验的结果一样, F-CMP 的吸附量比 $\mathrm{H}-\mathrm{CMP}$ 都有大幅度的提高, 对氯仿的吸附量最好, 达到 了 $43 \mathrm{~g} / \mathrm{g}$. 两个聚合物拥有相似的骨架结构, 但 F-CMP 由于氟元素的引入, 提高了材料的疏水性, 其对油和有 机溶剂都表现出了更高的吸附量.

为了进一步对材料的吸附性能进行表征, 我们测试 了材料的吸附速率和吸附循环性. 图 $4 \mathrm{e}$ 为聚合物对氯 仿的吸附量和时间的曲线, F-CMP 和 H-CMP 都只用 $10 \mathrm{~s}$ 的时间达到吸附饱和, 体现出快速的吸附速率. 图 S6 为 聚合物吸附氯仿的循环性的测试, 在经过 10 次循环以 后, F-CMP 和 H-CMP 的吸附量分别为 $35 \mathrm{~g} / \mathrm{g}$ 和 $15 \mathrm{~g} / \mathrm{g}$, 其中 F-CMP 的吸附量为初始的 $81 \%$, 表现出了更好的 


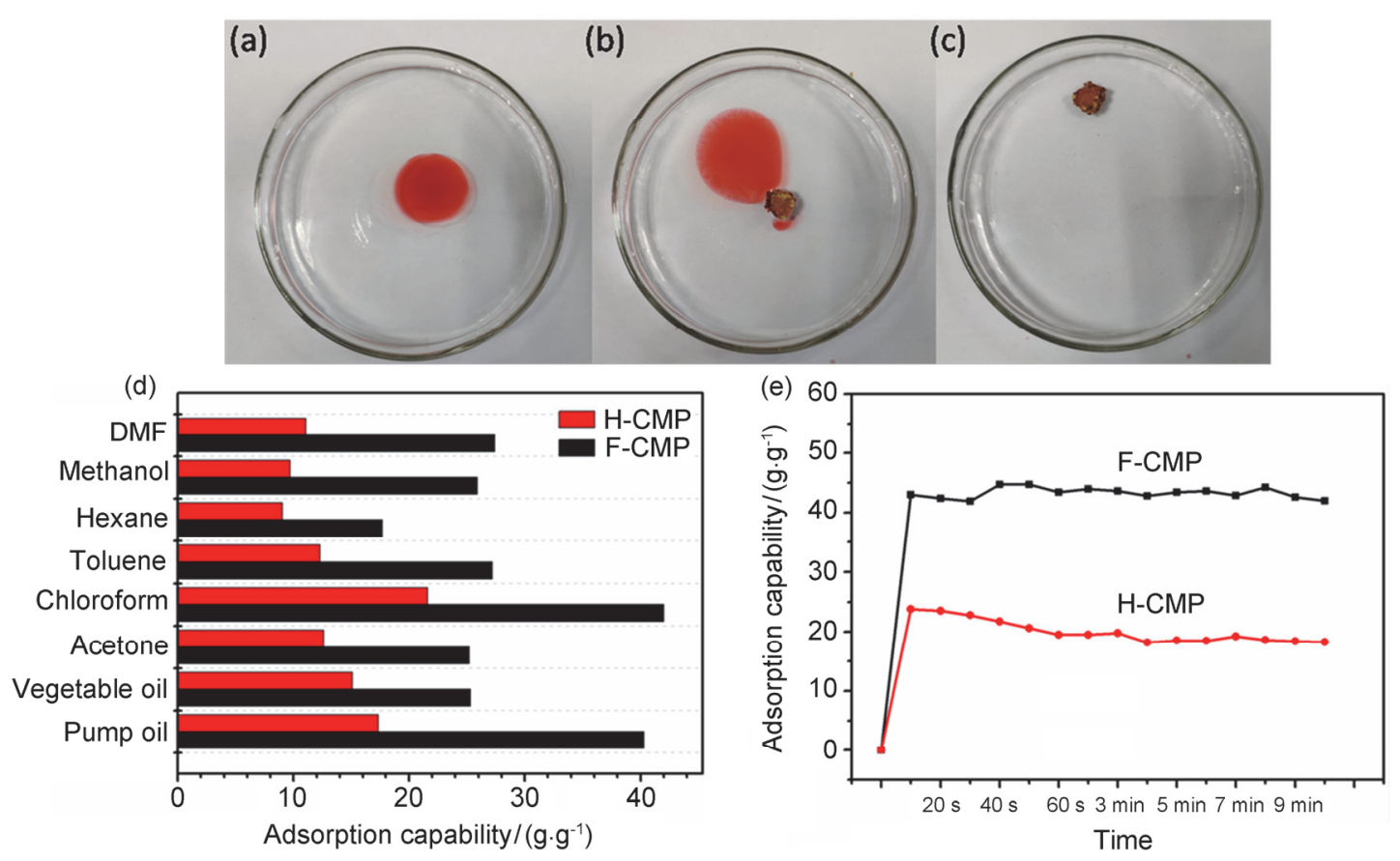

图 4 聚合物吸附甲苯(苏丹III染色)的过程 $(\mathrm{a} \sim \mathrm{c}$ ), 对于不同油和有机溶剂的吸附量 $(\mathrm{d})$, 聚合物对氯仿的吸附速率(e)

Figure 4 Adsorption process of toluene (dyed with oil-red III) ( $\sim$ c), Adsorption capacities for different solvents and oils (d), Instant adsorption capacity for chloroform

循环性能. 通过实验可以发现, F-CMP 对油和有机溶剂 的吸附性能较 H-CMP 有大幅度的提高, 主要由于 F-CMP 的骨架中引入了 $\mathrm{F}$ 原子, 提高了疏水性, 使材料 可以选择性吸附油和有机溶剂，同时也提高了吸附量. 其次 F-CMP 拥有更加合理的孔隙结构, 大量的微孔有 助于对溶剂的储存和吸附, 而介孔和大孔的存在不但可 以使溶剂快速地进入内部孔道中, 增加吸附速率, 而且 进一步增加聚合物和溶剂的接触面, 提高吸附量.

\section{3 结论}

选用 $1,3,5$-三氟-2,4,6-三碘苯作为含氟单体与 $1,3,5$ 三乙炔苯反应合成共轭微孔聚合物 F-CMP. 由于氟元素 的引入, F-CMP 的疏水性得到明显提高, 与水的接触角 达到 $145^{\circ}$. 氮气吸脱附测试显示 F-CMP 的比表面积达 到 $638 \mathrm{~m}^{2} \cdot \mathrm{g}^{-1}$ 且拥有更合理的孔隙结构, 有助于有机污 染物在聚合物中的传输和吸附. 得益于良好的疏水性能 和合理的孔隙结构, 相比于骨架结构相似的不含氟共轭 微孔聚合物, F-CMP 对油和有机溶剂的吸附量得到大幅 提高, 对原油和氯仿的吸附量达到最大, 分别为 $40 \mathrm{~g} / \mathrm{g}$ 和 $43 \mathrm{~g} / \mathrm{g}$, 且显示出高的吸附速率和良好的吸附循环性. 这些结果表明, 含氟共轭微孔聚合物是一类很有潜力的 水处理吸附材料.

\section{4 实验部分}

\section{1 单体 1,3,5-三氟-2,4,6-三碘苯(M2)的合成}

单体 1,3,5-三氟-2,4,6-三碘苯的合成方法参照文献
报道 ${ }^{[38]}$. 在 $0{ }^{\circ} \mathrm{C}$ 的条件下, 将高碘酸 $(342 \mathrm{mg}, 1.5 \mathrm{mmol})$ 溶于 $15 \mathrm{~mL}$ 的浓硫酸, 然后在搅拌状态下缓慢加入 KI (747 mg, $4.5 \mathrm{mmol}$ )粉末, 溶液呈黑色. 缓慢加入 1,3,5三氟苯 $(132 \mathrm{mg}, 1 \mathrm{mmol})$ 后, 撤掉冰水浴, 升温至 $70{ }^{\circ} \mathrm{C}$ 反应 $4 \mathrm{~h}$. 反应结束后, 产物缓慢倒入冰水浴中, 依次用 乙醚萃取, 饱和的 $\mathrm{NaHCO}_{3}$ 水溶液洗涤和无水 $\mathrm{Na}_{2} \mathrm{SO}_{4}$ 干燥. 通过减压旋蒸除去有机溶剂，柱层析分离得到白 色固体 $420 \mathrm{mg}$ (产率: 82\%).

\section{2 聚合物 H-CMP 的制备}

在无水无氧的条件下, 将 1,3,5-三乙炔苯 $(225 \mathrm{mg}$, $1.5 \mathrm{mmol}), 1,3,5$-三溴苯 $(315 \mathrm{mg}, 1 \mathrm{mmol}), \operatorname{Pd}\left(\mathrm{PPh}_{3}\right)_{4}(58$ $\mathrm{mg}, 0.05 \mathrm{mmol})$ 和 $\mathrm{CuI}(19 \mathrm{mg}, 0.1 \mathrm{mmol})$ 固体溶解于除 水的四氢呋喃 $(20 \mathrm{~mL})$ 和三乙胺 $(20 \mathrm{~mL})$ 的溶剂中, 升温 到 $80{ }^{\circ} \mathrm{C}$ 反应 $36 \mathrm{~h}$ 得到聚合物. 反应结束后降到常温, 把聚合物进行抽滤，并用水、甲醇、氯仿、丙酮等溶剂 洗涤，除去未反应的单体和催化剂. 得到的固体分别用 甲醇、氯仿和四氢呋喃索式提取 $24 \mathrm{~h}$, 真空干燥后得到 黄色固体 $217 \mathrm{mg}$ (产率: 96\%).

\section{3 聚合物 F-CMP 的制备}

聚合物 F-CMP 的合成方法和 H-CMP 合成方法类 似. 聚合反应中用到 1,3,5-三乙炔苯 $(225 \mathrm{mg}, 1.5 \mathrm{mmol})$, 1,3,5-三氟-2,4,6-三碘苯 $(510 \mathrm{mg}, 1 \mathrm{mmol})$ 单体, 以及 $\mathrm{Pd}\left(\mathrm{PPh}_{3}\right)_{4}(58 \mathrm{mg}, 0.05 \mathrm{mmol})$ 和 $\mathrm{CuI}(19 \mathrm{mg}, 0.1 \mathrm{mmol})$ 催 化剂. 聚合物在真空干燥后得到淡黄色块状固体 273 mg (产率: 99\%). 


\section{4 吸附实验}

材料吸附量的大小是通过聚合物吸附油和有机溶 剂前后的质量改变来确定的. 为了便于测试, 把聚合物 装入带有小孔的离心管中, 其中离心管管壁的小孔可以 保证油和有机溶剂的自由出入. 将离心管浸入油或者有 机溶剂中 $20 \mathrm{~min}$, 然后取出离心管, 去掉外管壁的油或 有机溶剂后进行称重. 吸附量的计算公式为:

$$
Q=\left(W_{\mathrm{t}}-W_{\mathrm{i}}\right) / W_{\mathrm{i}}
$$

其中, $Q$ 是聚合物的吸附量, $W_{\mathrm{i}}$ 是吸附前的聚合物的质 量 $(\mathrm{g}), W_{\mathrm{t}}$ 是吸附后的聚合物的质量 $(\mathrm{g})$.

\section{5 材料表征}

红外光谱由 Nicolet 560 型傅里叶变换红外光谱仪 测得. X 射线衍射谱图由 Philips X 'Pert PRO MPD 型 X 射线衍射仪测得, 扫描的范围为 $5^{\circ} \sim 80^{\circ}$. 热失重曲线 由 TG209F1 型号热重分析仪测得, 采用 $2 \sim 10 \mathrm{mg}$ 样品 在氮气氛围下以 $10{ }^{\circ} \mathrm{C} / \mathrm{min}$ 的升温速率从 $30{ }^{\circ} \mathrm{C}$ 升温到 $800{ }^{\circ} \mathrm{C}$ 进行测试. 形貌表征采用日本 JEOL JSM-7500F 型扫描电子显微镜. 气体吸附实验采用日本拜耳公司的 BELSORB Max 吸附分析仪. 在 $77 \mathrm{~K}$ 的条件下, 测试聚 合物对 $\mathrm{N}_{2}$ 的吸附和解吸附. 孔径分布曲线通过使用吸 附等温线的非定域密度泛函理论(NL-DFT)模拟得到. X 射线光电子能谱分析采用 XSAM 800 型号仪器测试.

\section{References}

[1] Shannon, M. A.; Bohn, P. W.; Elimelech, M.; Georgiadis, J. G.; Marinas, B. J.; Mayes, A. M. Nature 2008, 452, 301.

[2] Doshi, B.; Sillanpaa, M.; Kalliola, S. Water Res. 2018, 135, 262.

[3] Gupta, S.; Tai, N. H. J. Mater. Chem. A 2016, 4, 1550.

[4] Lei, E.; Li, W.; Ma, C.; Liu, S. Mater. Chem. Phys. 2018, 214, 291.

[5] Mohan, D.; Singh, K. P.; Singh, V. K. J. Hazard Mater. 2008, 152, 1045.

[6] Sakthivel, T.; Reid, D. L.; Goldstein, I.; Hench, L.; Seal, S. Environ. Sci. Technol. 2013, 47, 5843.

[7] Likon, M.; Remskar, M.; Ducman, V.; Svegl, F. J. Environ. Manage. 2013, 114, 158.

[8] Zhang, T.; Kong, L.; Dai, Y.; Yue, X.; Rong, J.; Qiu, F.; Pan, J. Chem. Eng. J. 2017, 309, 7.

[9] Chin, S. F.; Binti, R. A. N.; Pang, S. C. Mater. Lett. 2014, 115, 241.

[10] Ong, C. C.; Sundera, M. S.; Mohamed, N. M.; Perumal, V.; Mohamed, S. M. S. ACS Omega. 2018, 3, 15907.

[11] Ji, C.; Zhang, K.; Li, L.; Chen, X.; Hu, J.; Yan, D.; Xiao, G.; He, X. J. Mater. Chem. A 2017, 5, 11263.
[12] Peng, D.; Jiang, W.; Li, F. F.; Zhang, L.; Liang, R. P.; Qiu, J. D. ACS Sustainable Chem. Eng. 2018, 6, 11685.

[13] Dawson, R.; Laybourn, A.; Clowes, R.; Khimyak, Y. Z.; Adams, D. J.; Cooper, A. I. Macromolecules 2009, 42, 8809.

[14] Dawson, R.; Cooper, A. I.; Adams, D. J. Prog. Polym. Sci. 2012, 37, 530.

[15] Yan, T. T.; Xing, G. L.; Ben, T. Acta Chim. Sinica 2018, 76, 366. (闵 婷婷, 邢国龙, 贲腾, 化学学报, 2018, 76, 366.)

[16] Pang, C.; Luo, S. H.; Hao, Z. F.; Gao, J.; Huang, Z. H.; Yu, J. H.; Yu, S. M.; Wang, Z. Y. Chin. J. Org. Chem. 2018, 38, 2606. (庞楚明, 罗 时荷, 郝志峰, 高健, 黄召昊, 余家海, 余思敏, 汪朝阳, 有机化 学, 2018, 38, 2606.)

[17] Yu, S.; Xu, Y. J.; Jiang, J. X.; Ren, S. J. Acta Chim. Sinica 2015, 73, 629. (于森, 徐雍捷, 蒋加兴, 任世杰, 化学学报, 2015, 73, 629.)

[18] Xu, Y.; Jin, S.; Xu, H.; Nagai, A.; Jiang, D. Chem. Soc. Rev. 2013, 42,8012 .

[19] Jiang, J. X.; Su, F.; Trewin, A.; Wood, C. D.; Campbell, N. L.; Niu, H.; Dickinson, C.; Ganin, A. Y.; Rosseinsky, M. J.; Khimyak, Y. Z.; Cooper, A. I. Angew. Chem. Int. Ed. 2007, 46, 8574.

[20] Chen, Q.; Liu, D. P.; Luo, M.; Feng, L. J.; Zhao, Y. C.; Han, B. H. Small 2014, 10, 308.

[21] Wang, X.; Chen, B.; Dong, W.; Zhang, X.; Li, Z.; Xiang, Y.; Chen, H. Macromol. Rapid Commun. 2018, e1800494.

[22] Kong, S. N.; Malik, A. U.; Qian, X. F.; Shu, M. H.; Xiao, W. D. Chin. J. Org. Chem. 2018, 38, 656. (孔胜男, Abaid Ullah Malik, 钱 雪峰, 舒谋海, 肖文德, 有机化学, 2018, 38, 656.)

[23] Kong, S. N.; Qian, X. F.; Shu, M. H.; Xiao, W. D. Chin. J. Org. Chem. 2018, 38, 2754. (孔胜男, 钱雪峰, 舒谋海, 肖文德, 有机 化学, 2018, 38, 2754.)

[24] Xu, Y. J.; Wu, S. P.; Ren, S. J.; Ji, J.; Yue, Y.; Shen, J. J. RSC $A d v$ 2017, 7, 32496

[25] He, Q.; Zhang, C.; Li, X.; Wang, X.; Mu, P.; Jiang, J. X. Acta Chim. Sinica 2018, 76, 202. (贺倩, 张崇, 李晓, 王雪, 牟攀, 蒋加兴, 化 学学报, 2018, 76, 202.)

[26] Liao, Y.; Cheng, Z.; Zuo, W.; Thomas, A.; Faul, C. F. J. ACS Appl. Mater. Interfaces 2017, 9, 38390.

[27] Bildirir, H.; Osken, I.; Ozturk, T.; Thomas, A. Chemistry 2015, 21, 9306.

[28] Qiu, F.; Zhao, W.; Han, S.; Zhuang, X.; Lin, H.; Zhang, F. Polymers 2016, 8, 191.

[29] Yang, R. X.; Wang, T. T.; Deng, W. Q. Sci. Rep. 2015, 5, 10155.

[30] Lee, J. S. M.; Wu, T. H.; Alston, B. M.; Briggs, M. E.; Hasell, T.; Hu, C. C.; Cooper, A. I. J. Mater. Chem. A 2016, 4, 7665.

[31] Qin, L.; Xu, G. j.; Yao, C.; Xu, Y. Polym. Chem. 2016, 7, 4599.

[32] Cha, M. C.; Lim, Y.; Choi, T. J.; Chang, J. Y. Macromol. Chem. Phys. 2017, 218, 1700219.

[33] Shen, X.; He, J.; Wang, K.; Li, X.; Wang, X.; Yang, Z.; Wang, N.; Zhang, Y.; Huang, C. ChemSusChem 2019, 12, 1342.

[34] Zhao, X.; Wu, M.; Liu, Y.; Cao, S. Org. Lett. 2018, 20, 5564.

[35] Ju, Z.; Zhang, S.; Xing, Z.; Zhuang, Q.; Qiang, Y.; Qian, Y. ACS Appl. Mater. Interfaces 2016, 8, 20682.

[36] Wang, X.; Mu, P.; Zhang, C.; Chen, Y.; Zeng, J.; Wang, F.; Jiang, J. X. ACS Appl. Mater. Interfaces 2017, 9, 20779.

[37] Xu, L.; Xiao, G.; Chen, C.; Li, R.; Mai, Y.; Sun, G.; Yan, D. J. Mater. Chem. A 2015, 3, 7498 .

[38] Wenk, H. H.; Sander, W. Eur. J. Org. Chem. 2002, 3927. 